\title{
Characteristics of In-Memory Business Intelligence
}

\author{
Mihaela-Laura IVAN \\ Department of Economic Informatics and Cybernetics \\ Bucharest University of Economic Studies \\ ivanmihaela88@gmail.com
}

Business Intelligence (BI) is an important key in the initiatives of many organizations. BI has developed over the years and the most businesses need to take it to the next level of maturity and improvement can be enabled by SAP HANA. In this paper I presented the evolution of Business Intelligence industry, what others capabilities enterprises still seek and how Business Warehouse on SAP Hana enable these needs. Also, the paper highlights that Inmemory Business Intelligence is powerful than data warehouse and Online Analytical Processing projects.

Keywords: Business Intelligence, Data Warehouse, Optimization, SAP HANA, In-Memory

1 Introduction

Business Intelligence, or BI, is essential to the success of companies because the ability to process a vast amount of data from a large range of sources anywhere and anytime it's decisive. The analyzed technology is used to make data accessible through: cost-cutting suppositions, expose extraordinary business opportunities, transfer ERP data into reports, react fast to retail demand and optimize [1]. Another important BI utilization is to find those inefficient business processes. This led to responsiveness in fast improvements for future growth. For example, a group of people used BI software to identify opportunities on how to reduce mobile phone usage overtime, saving $\$ 2$ million rapidly. That is why BI is more useful when it is used to uncover wicked business problems than when it is used to monitor what's happening [2].

\section{The Importance of Business Intelligence} Business Intelligence becomes a key strategy for most of the big organizations for achieving a competitive advantage. Forasmuch, during the past ten years, the approach to management business in the whole word has changed profoundly, organizations started to be able through knowledge and data conversion, shaping the correct information in short periods of time [3]. Thus, development of BI segment within organizations becomes an elementary concept for most of the managers in order to increase this function within business and evaluate their performances using BI tools and Data Warehouse Applications. BI systems are instruments with the help of which the results of a company can be estimated [4]. It was demonstrated so far that organizations which make use of BI tools for operating at decision level are more competitive and can evaluate the business in a more realistic way. This means that cybernetic places like big enterprises and organizations are more easily to be managed in the last years like they were before. If we analyze more deeply this topic, we can simple realize that this is a cause of the global cybernetic evolution regarding informatics industry.

Time became so far a resource which had to be optimized, rationalized and controlled within an organization [5]. Thus, having tools which can help decision level to take decisions and manage the human resources could be another advantage for growing in a competitive environment. In the course of history, people perception regarding passed time was undergone to changes. Now we can observe that they realize that decreasing period of work for most of employees and the rhythm of activities also could go in a next era of evolution and civil organization.

Giving the fact that this concept of optimizing activities within organizations 
becomes more and more popular, experts attention headed to defining and explaining most important characteristics with the final scope to improve, demonstrate and also develop further this market industry. Taking a large overview on most essential features, we can delimit the following three quality characteristics:

- Easy of use: End-users can easily use BI reports because they are quite natural and helps in interpreting data, having in most cases the possibility of causing a drill-down. The final output of BI tool is in most often cases very easy to interpret and managed as final information for decision and organizational levels.

- Flexibility: This means that BI applications can be accessed and utilized on multiple kinds of operating systems, generated by Linux or Windows trademark, being also able to be moved and extended from one version to another. These can also be designed by every client in a simplistic form or even created. Flexibility is also covered by the configurable propriety of most of the BI reports.

- Data security: Servers for storing Business Objects have encrypted database to secure information related directories, reports, users, universes. Big providers of BI tools uses specialized encryption algorithms for fulfil this big requirement, implemented by specialized experts.

Taking into account that BI applications were first designed in 2011 and that the requirements of BI Reports became a new topic for many companies, for example SAP provided documentations and specialized books for their employees for creating BI reports and application tools. This is a big insurance for most of the young developers which want to straighten to this niche they have materials and have the capabilities for understanding and predicting that their further activities will grow fast in this industry. The vision well seen by the most of big CEO's and managers from big organizations is to assure and recruit young professional with deep knowledge and talent for this BI aptness, also to encourage any new big idea for extending the industry.

\section{Business Intelligence Trends on the Global Market}

Gartner predicted that in 2014 Business Intelligence will be CIO's Technology Priority. In the period 2013-2018 was said that BI market will grow with 9 percent [6]. This happens because the volume of information in almost all industries was increasing. The challenge for BI evolution of this market is the high costs of BI applications implementation.

The most important worldwide BI experts have been expressed their visions regarding Business Intelligence trends in 2014. These latest ideas can help users to improve and predict some of the further evolution of the industry, in order to optimize daily work activities for developing BI tools and discover the latest market trends described below [7]:

- Equilibrium between demand and supply - BI Tools still remain the most important niche due to the forecast that vendors made for maturing BI software market and improve strategies with customers.

- Excellent development skills - Young developers which want to learn to improve BI tools must first have strong development skills regarding enterprise systems for transactional and operational data and must first know how to interpret this data. This should be a big advantage in growing and developing BI tool in this business.

- Understanding the utility of BI tools It is became critical to sustain investment in user education, as this is the key for possessing fundamental knowledge about how they can support Business Intelligence tools.

- Mobile and Cloud BI Services for optimizing activities - Those who use cloud BI services need to have a different mindset for measuring BI area in new way. 
- Developing BI Businesses independently from traditional IT Industry - The major trend in BI evolution will be the business problems. In doing that, once the business solutions is captured for inmemory reporting without an army of coders anything can happen.

- Evolution, expansion and development of BI Industry - These indicators will remain positive in the next period and it can exist the possibility to embed BI tools in other hardware, software and equipment, which will cause the expansion and growth of the business.

\section{In-Memory Business Intelligence}

The in-memory Business Intelligence incorporates a high-performance by providing important benefits as: accelerate analytics with data modelling changing from mandatory to optional activity, significantly reduce planning cycles by processing calculations in-memory. This market leading platform avoid exploding data volumes that limits visibility and reduce wait times for reports so the business community can react in the moment. A high performance Business Intelligence enables businesses to analyze data volumes fast. These are the main reasons why real-time data platform makes the businesses react fast. In-memory Business Intelligence (BI) makes reference to Business Intelligence software that utilizes in-memory database (IMDB).

In-memory database is a database management system that is designed for best performance with condition to exist enough computer memory to hold the needed data. This is in contrast to Relational Database Management Systems which is designed also for best performance. [8] "You need at least eight hours currently before data is available in the BI tools associated with ERP," said SAP's Klein. While in-memory BI reduces this delay from hours to few seconds". [9] Inmemory databases promise a faster analysis of large amounts of data. What is the potential for this technology or whether it really is a new paradigm for Business
Intelligence solutions, it is highlighted in this article.

"In-memory" sounds optimistic since the announcement of the SAP HANA product in mid-2011 in the IT market. In comparison with the cloud computing, the benefit to the user is immediately understandable, because it comes from faster analysis of large data sets. In-memory databases are the future of Business Intelligence, said the leading experts like Wolfgang Martin from Wolfgang Martin Team and Rüdiger Spies, vice president of IDC Central Europe and not only them were seeing the great potential of the new technology with the features of analytical and operational business applications.

In fact, what is new in-memory BI? Because of the general enthusiasm seems a critical look at the real facts. What does happen with in-memory? What are the differences between in-memory database (IMDB) and traditional relational database management systems (RDMS)? Answer to these questions is given after a look at the development of the processor and memory technologies in the last recent years. In the same measure in which the processing speed of processors grew, the access times of conventional hard disk storage systems become a bottleneck for the data flow. Also, multicore CPUs for parallel processing of data in memory supports this process. In sum, these technical innovations form the basis for a new way of working of business applications, particularly databases. In contrast to conventional relational databases the in-memory databases don't need have the data to be processed from a hard drive or loading it into memory, because the data is already there. This shortens the response time of the database dramatically and acts as a turbo for the corresponding applications.

The usage of In-memory Business Intelligence in SAP Fiori is presented below. SAP Fiori UX is the new face of SAP to business users in all lines of business across devices and deployment options. It provides optimal usability for simplified business interactions and ease of use. SAP solutions, 
such as our SAP Business Suite powered SAP HANA, are applying the SAP Fiori UX to provide a personalized, responsive and simple user experience. SAP Fiori UX increases user satisfaction with SAP software by providing a delightful user experience. SAP Smart Business cockpits, which apply SAP Fiori UX, empower employees with instant insight-to-action - allowing access to real-time information and the ability to view KPIs and complete transactions in the same process. SAP Fiori allows employees to work seamlessly across devices - desktop, tablet, or smartphone. It provides improved user satisfaction and enables quicker approvals and better decisions. SAP FIORI is an application developed using SAP HANA and SAP UI5. [10]

The real-time data platform from SAP is the next generation database. This database provides advantages such as efficient interprocess communication taking place inside one overall system, same data types, etc. provide performance advantages that no other application server can match when SAP HANA is the database. The SAP HANA inmemory computing is used to simplify and to benefit from the in-memory process innovation in Business Intelligence domain [11].

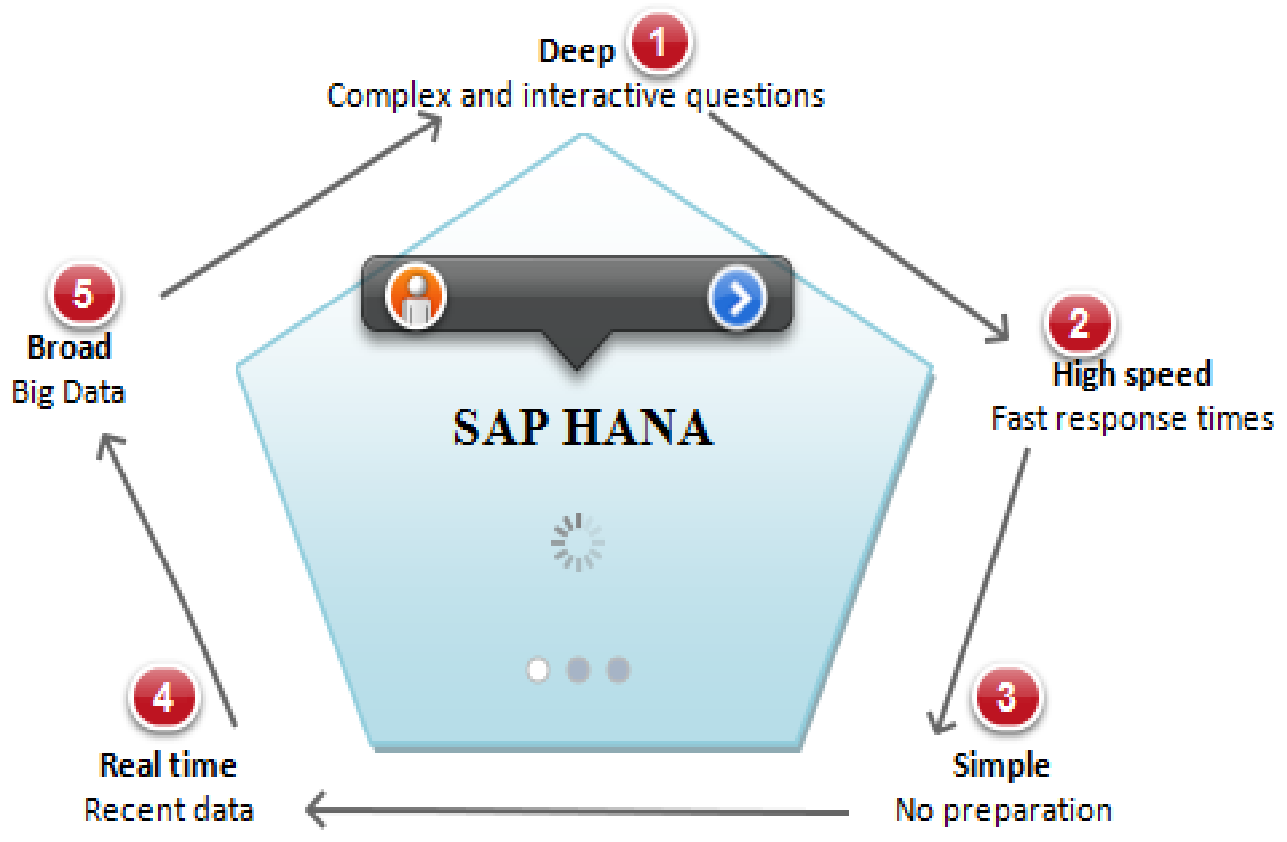

Fig. 1. The SAP HANA in-memory database platform

Nowadays, many people within an organization need to analyze big data in real time and to have its insights available as fast as possible. In other cases, the customers are looking for long-term options. That is why they are considering leveraging more value out of investments. They are looking at better and faster planning and reporting applications. Business Warehouse on SAP HANA is the Modern Data Warehouse. SAP BW powered by SAP HANA is a strategic solution for most Enterprise Data Warehouse scenarios like real-time analytics, big data volumes or in-memory computing. The key features provided by the SAP HANA inmemory database platform presented in Figure 1 makes possible to have faster time for production, accelerated deployment [12]. In the Berlin Charité is in-memory technology already being used for the improvement of cancer therapies. The practical benefits of in-memory databases were illustrated with an application example in the Berlin Charité. Therefore, the Charité Medicine, Charité-IT, the SAP Innovation Center Potsdam and the Hasso Plattner Institute (HPI), the research initiative "HANA Oncolyzer" established to develop 
the IT-based treatment for cancer patients. The better knowledge about tumors and drugs active ingredients will help even more closely cancer treatments for patients. In this way will be improved faster the chances of recovery. Based on the technology SAP inmemory computing can be used the HANA Oncolyzers even for large amounts of data in very short time. With the help of these technologies the tumor cell genomes of all cancer patients will be decrypted and examined in detail in order to allow individual therapies. This results are per patient data volumes which must be searched and analyzed for successful treatment possible in real time according to the relevant mutation information. With the efforts of Hasso Plattner Institute (HPI) and the SAP Innovation Center Potsdam was developed the prototype HANA Oncolyzer. Even companies already use HANA. The Austrian company Red Bull for example, uses the solution to manage the business with cuttingedge data from its offices in more than 160 countries. The German stock exchange meets its need for in-memory computing on the other hand with Oracle Times Ten [18]

\section{The role of Business Warehouse on SAP HANA in the future of Business Intelligence}

The work done in the real-time analytics, inmemory database, with memory becoming cheaper, multi- core architecture which allow parallel processing, compression techniques useful to keep more data in less memory, column and row storage being able to access data at amazing speed and real-time replication capability of data is the role of SAP HANA. These capabilities are illustrated in Figure 2 [13].

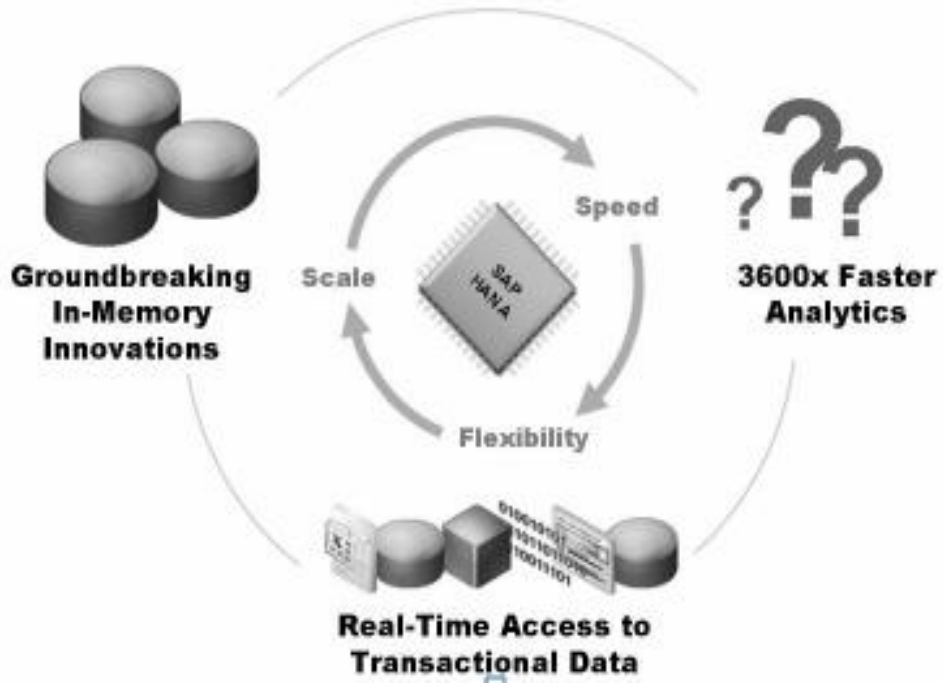

Fig. 2. The key capabilities of SAP HANA

In light of the aspects I presented above, I would like to turn my attention to SAP company which has revealed new technological innovations with Data Warehousing. SAP NetWeaver Business Warehouse (BW) is the name of Business Intelligence (BI).

Business Warehouse on SAP HANA is the Modern Data Warehouse. SAP BW powered by SAP HANA is a strategic solution for most Enterprise Data Warehouse scenarios like real-time analytics, big data volumes or in-memory computing [14].

Nowadays, many people within an organization need to analyze big data in real time and to have its insights available as fast as possible. Although, the customers are looking for long-term options. That is why they are considering to leverage more value out of investments. They are looking at better and faster planning and reporting applications. 
In Figure 3 are presented the main important HANA technology [15]. challenges, factors and benefits of $\mathrm{BW}$ on
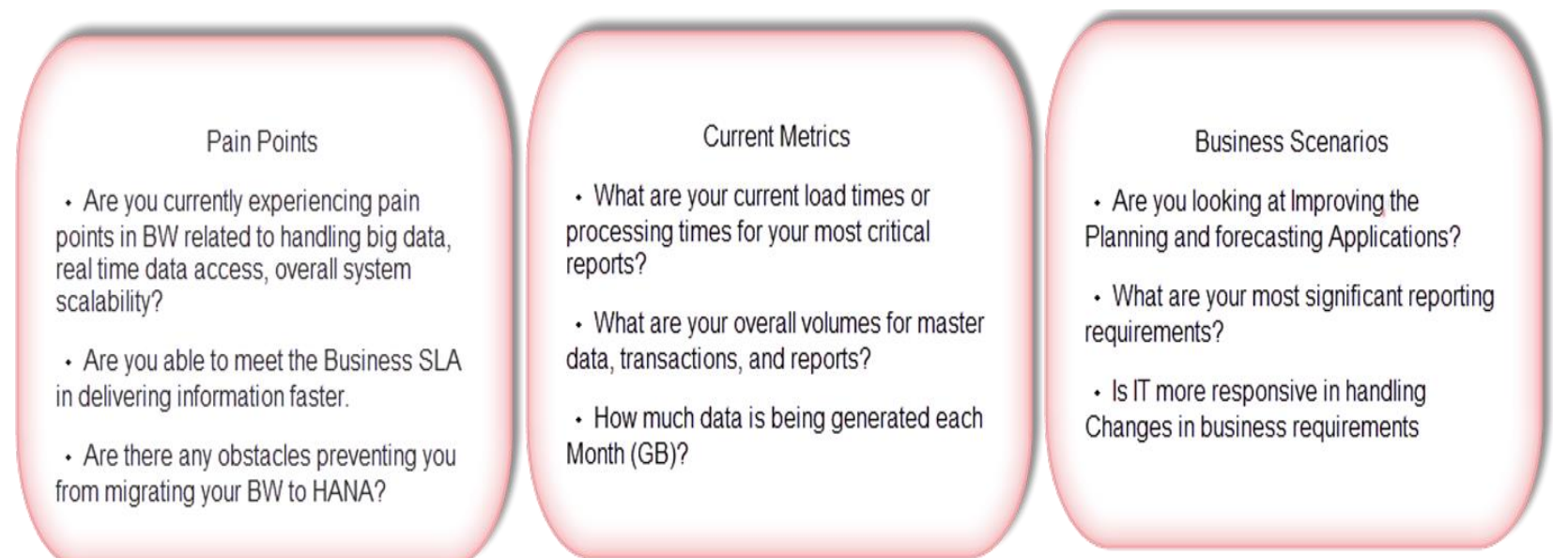

Fig. 3. Challenges, factors and benefits of BW on HANA

As in each renewal it's important to estimate the overall advantages and disadvantage. The above qualification questions address the key attributes to find if BW on HANA technology is the right choice for their businesses. These are necessary to make the customers aware of BW on HANA need, to identify where people can take advantage of it and to emphasize on how their organization can benefit [16].

\section{The comparison of OLAP and in- memory Business Intelligence}

A comparison between these two Business Intelligence concepts on the basis of: its architecture, their construction or expansion, their planning functions, how to choose in terms of your requirements the right Business Intelligence technology in less time.

\subsection{Comparison based on architecture}

If we take into consideration Business Intelligence architectures; this is basically for both Business Intelligence procedure similar. The structure and the handling of in-memory architecture is somewhat simpler. Another distinguishing factor is that modern OLAP databases are more open than the in-memory systems in general, since they can be integrated from the outside via MDX query. Also in OLAP, a complex two-stage loading (ETL to OLAP DB) is required. This may be an area that exceeds the needs and knowledge of the users.

However, both Business Intelligence architectures have limitations for very large amounts of data, which are based on the availability of the server.

\subsection{Comparison based on expansion}

Both Business Intelligence infrastructures are basically simple and fast. Product training is necessary for the introduction of the respective Business Intelligence software / platform. In OLAP skills and experience in modeling are required. In addition, care should be taken in addition to the database exactly on the included Business Intelligence tools. OLAP DB and Excel Viewer are part of the software package.

The Business Intelligence technology "inmemory" is usually easily extendable. That means that new data can be easily integrated. An initial deployment, however, should be supervised by an experienced specialist.

\subsection{Comparison based on the planning functionality}

Modern OLAP databases have the option for planning. Some OLAP manufacturer are specialized, but on different planning functions like TM1 and Infor. The planning requirements should be analyzed in more detail in advance and verified in a proof of concept. Although this Business Intelligence 
technology can assist in planning tasks, it The following table summarizes the features does not replace a planning system. In of the Business Intelligence process together: memory technology usually does not offer planning functionality.

Table 1. The comparison of OLAP and in-memory Business Intelligence

\begin{tabular}{|c|c|c|}
\hline Business Intelligence Method & OLAP & In Memory \\
\hline \multicolumn{3}{|l|}{ Architecture } \\
\hline $\begin{array}{l}\text { Facilitating the structure and handling of } \\
\text { the architecture }\end{array}$ & $-->$ & $-->$ \\
\hline OLAP processing & $-->$ & $-->$ \\
\hline $\begin{array}{l}\text { From the outside via MDX query } \\
\text { integrable (openness) }\end{array}$ & $-->$ & $<-$ \\
\hline Easy to load without special knowledge & 0 & $-->$ \\
\hline Limits for very large amounts of data & $<--$ & $<--$ \\
\hline \multicolumn{3}{|l|}{ Building and expansion } \\
\hline Implement simple and fast & 0 & $-->$ \\
\hline Necessity to note the tools supplied & $<--$ & 0 \\
\hline Easily extendable & 0 & $-->$ \\
\hline \multicolumn{3}{|l|}{ Planning functionality } \\
\hline Write back & $-->$ & $<--$ \\
\hline Planning functions & $-->$ & $<--$ \\
\hline
\end{tabular}

\section{Conclusions}

In this paper, were presented the characteristics of Business Intelligence beyond data warehouse and Online Analytical Processing. Business Intelligence is used to improve decision making, cut costs and discover new business opportunities. [17]
The most important word in Business Intelligence area is data. Because enterprise data is expected to grow $650 \%$ in the near future customers benefit from the HANA opportunity. BW on HANA, the new generation of Business Intelligence released by SAP, helps customers realize value faster with less risk. Some clients declared that they see an extraordinary opportunity to improve 
their enterprise data warehouse solutions with SAP NetWeaver BW powered by SAP HANA. The strategic SAP approach provides the quickest time to value at a predictable price to achieve the desired benefits resulting from a full adoption of HANA. SAP HANA is an in-memory database platform that allows the processing of massive quantities of real time data - delivered as an appliance. For a business, this means to make value of the following advantages: react and analyze in real-time, make operation simplified, have a business visibility, drive business value from analyzed data.

\section{Acknowledgement}

This paper was co-financed from the European Social Fund, through the Sectorial Operational Programme Human Resources Development 2007-2013, project number POSDRU/159/1.5/S/138907 "Excellence in scientific interdisciplinary research, doctoral and postdoctoral, in the economic, social and medical fields -EXCELIS", coordinator The Bucharest University of Economic Studies

\section{References}

[1] M. L. Ivan, A. Pascal, "The future of Business Intelligence," Proceedings of the 13th International Conference on Informatics in Economy, IE 2014, May 15-18, 2014, Bucharest, Romania, ASE Printing House, ISSN 2284-7472, ISSNL 2247-1480.

[2] R. Mulcahy. (2013, November 13). Business Intelligence Definition and Solutions, CIO magazine [Online]. Vol. 1, No. 4, Available at: http://www.cio.com/article/40296/Busin ess_Intelligence_Definition_and_Soluti ons?, page $=3 \&$ taxonomyId $=3002$.

[3] A. Fereydoon, A. M. Mohammad, "Business Intelligence as a key strategy for development organizations", Proceedings of First World Conference on Innovation and Computer Sciences, Vol. 1, 2012, pp. 102-106.

[4] L. Serbanescu, M. Radulescu, "Optimizing time in business with Business Intelligence solution",
Proceedings of World Conference on Business, Economics and Management, Vol. 62, Antalya, Turkey, October 24, 2012, pp. 638-648.

[5] D. A. Berta,"Study Regarding the Perception of the Concept of Business Intelligence Among Application", Proceedings of 2nd World Conference On Business, Economics And Management, Dubai, United Arab Emirates, 2014, no.109, pp. 402-406.

[6] T. Priyank (2014, March 7), "Global Business Intelligence (BI) Market in Retail Industry 2014 - 2018" [Online], Available:

http://www.prweb.com/pdfdownload/11 446898.pdf.

[7] R. Drew (2014, January 2), "Top Business Intelligence Trends For 2014" [Online], Available: http://www.enterpriseappstoday.com/bus iness-intelligence/top-businessintelligence-trends-for-2014.html.

[8] What Is In-Memory BI?, Available at: http://www.sisense.com/what-is-inmemory-bi/

[9] D. Robb, In-Memory Analytics Buyer's Guide: Oracle Big Data/Exalytics Appliances vs. SAP HANA, Available at:

http://www.enterpriseappstoday.com/bus iness-intelligence/in-memory-analyticsbuyers-guide-oracle-big-dataexalyticsappliances-vs.-sap-hana.html

[10] SAP Fiori - Architecture overview, Available at: http://www.sdn.sap.com/irj/scn/go/portal /prtroot/docs/library/uuid/e03be70d68fb-3010-c6a0ff56889c2c81?QuickLink=index \&overri delayout=true $\& 58652073478233$

[11] Supercharge your data warehouse while simplifying IT - with SAP HANA inmemory computing, Available at: http://www.sap.com/pc/tech/in-memorycomputing-hana/software/analytics/datawarehouse.html

[12] M. L. Ivan, "Improving decision making process through Business Intelligence technology," Proceedings of 2nd 
International Conference 'Economic Scientific Research - Theoretical, Empirical and Practical Approaches', ESPERA 2014, (in progress), November 13-14, 2014, Bucharest, Romania.

[13] SAP AG or an SAP affiliate company, SAP HANA Introduction,

Participant Handbook, 2013, pp. 2-18.

[14] DC READINESS SAP HANA. Building a Trusted SAP HANA Data Center. Internet:

http://www.cisco.com/assets/events/i/sap te-hana_whitepaper.pdf, September 2013.

[15] SAP AG or an SAP affiliate company, SAP HANA Implementation and
Modeling, Participant Handbook，2013, pp. 176-180.

[16] R. Silberstein, Introducing...SAP HANA Extended Application Services (XS), Available at: http://www.saphana.com/docs/DOC3789

[17] M. Golfarelli, S. Rizzi, I. Cella Beyond Data Warehousing: What's Next in Business Intelligence?, Available at: http://www.cci.drexel.edu/faculty/song/d olap/dolap04/paper/p1-rizzi.pdf

[18] R. Richter, HANA Speeds Cancer Research and Results, Available at: http://scn.sap.com/community/hana-inmemory/blog/2012/04/04/hana-speedscancer-research-and-results

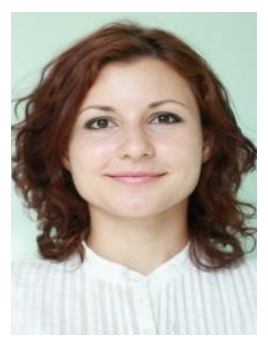

Mihaela Laura IVAN graduated from the Faculty of Cybernetics, Statistics and Economic Informatics of the Bucharest University of Economic Studies in 2011. She also finished the Master's degree in Economic Informatics in 2013, at the Bucharest University of Economic Studies. Starting with 2013, Mihaela is a PhD candidate at Bucharest University of Economic Studies in the field of Economic Informatics. At the present, she is a SAP Development Consultant at SAP Near Shore Center Romania. 